\title{
ON THE LAGUERRE METHOD FOR SEPARATING THE ROOTS OF ALGEBRAIC EQUATIONS ${ }^{1}$
}

STEPHEN KULIK

1. Introduction. The celebrated French mathematician E. N. Laguerre gave a proposition [2] which says that any two numbers $u$ and $v$ satisfying the relation

$$
(u-x)(v-x)\left(f^{\prime 2}-f f^{\prime \prime}\right)+(u+v-2 x) f f^{\prime \prime}+N f^{2}=0,
$$

where $f \equiv f(x), f(x)=0$ is an algebraic equation of degree $N$ with real roots and $x$ an arbitrary real number, separate the roots of the equation. This proposition was also discussed by $\mathrm{Ch}$. Hermite [2] and by E. Bodewig [1].

According to the proposition there will be at least one root of the equation between $u$ and $v$. A generalization of the relation (1), given in the following section gives a method for separating just one root and, in fact, to evaluate it.

2. A method for separating the roots of algebraic equations. Let $f(x)=0$ be an algebraic equation with real roots $a_{i}, i=1,2, \cdots, N$. Consider the expansion of the function $(u-x)(v-x) f^{\prime}(x) / f(x)$ into the partial fractions

$$
\begin{aligned}
& (u-x)(v-x) f^{\prime}(x) / f(x) \\
& \quad=\sum\left(u-a_{i}\right)\left(v-a_{i}\right)\left(x-a_{i}\right)^{-1}+N(x-u-v)+\sum a_{i},
\end{aligned}
$$

where the sum is taken over all the roots of the equation. After differentiating $n-1$ times both sides of the equation (2) and dividing by $(n-1) !(-1)^{n-1}$, we obtain

$$
\begin{aligned}
{\left[(u-x)(v-x) D_{n}+\right.} & \left.(u+v-2 x) f D_{n-1}+f^{2} D_{n-2}\right] f^{-n} \\
& =\sum\left(u-a_{i}\right)\left(v-a_{i}\right)\left(x-a_{i}\right)^{-n} \quad n \geqq 3,
\end{aligned}
$$

where $f \equiv f(x)$, and

$$
D_{n}=\left|\begin{array}{ccccc}
f^{\prime} & f & 0 & \cdots & 0 \\
f^{\prime \prime} & f^{\prime} & f & \cdots & 0 \\
\cdot & \cdot & \cdot & \cdots & 0 \\
\frac{f^{(n)}}{(n-1) !} & \frac{f^{(n-1)}}{(n-1) !} & \frac{f^{(n-2)}}{(n-2) !} & \cdots & f^{\prime}
\end{array}\right|
$$

Presented to the Society, August 22, 1956; received by the editors November 19, 1956 and, in revised form, February 15, 1957.

1 Sponsored by the Office of Ordnance Research, U. S. Army. 
$D_{n}$ is evaluated recursively, namely

$$
\begin{aligned}
D_{n}= & f^{\prime} D_{n-1}-f^{\prime \prime} f D_{n-2} / 2 !+\cdots+(-f)^{n-2} f^{(n-1)} D_{1} /(n-1) ! \\
& +(-f)^{n-1} f^{(n)} D_{0} /(n-1) !,
\end{aligned}
$$

where the second term will be $-f^{\prime \prime} f$ when $n=2$.

Thus, if we fix $x \neq a_{i}$, the solutions of the equation

$$
(u-x)(v-x) D_{n}+(u+v-2 x) f D_{n-1}+f^{2} D_{n-2}=0
$$

coincide with the solutions of the equation

$$
\sum\left(u-a_{i}\right)\left(v-a_{i}\right)\left(x-a_{i}\right)^{-n}=0 .
$$

Now, if $n$ is even and $x$ an arbitrary real number, then $u$ and $v$ which satisfy the equation (7) would be divided by at least one root of the equation $f(x)=0$.

Further, if

$$
\left|x-a_{1}\right|<\left|x-a_{i}\right|, \quad i=2,3, \cdots, N
$$

then

$$
\lim _{n \rightarrow \infty} u(n)=a,
$$

where $u(n) \equiv u, v$ is any fixed real number, and $a \equiv a_{1}$ represents any root of the equation $f(x)=0$.

Solving now equation (6) for $u$ we have the result

$$
\begin{array}{r}
u=x-f\left[(v-x) D_{n-1}+f D_{n-2}\right] /\left[(v-x) D_{n}+f D_{n-1}\right], \\
n=4,6, \cdots
\end{array}
$$

and

$$
a=x-\lim _{n \rightarrow \infty} f\left[(v-x) D_{n-1}+f D_{n-2}\right] /\left[(v-x) D_{n}+f D_{n-1}\right] .
$$

Two particular cases are worth noting:

$$
u=x-f D_{n-2} / D_{n-1}, \quad \text { when } v=x,
$$

and

$$
u=x-f D_{n-1} / D_{n}, \quad \text { when } v=\infty .
$$

The Laguerre case can be obtained from (10) by taking $n=2$ and substituting $N$ for $D_{0}$; however, $D_{2}$ and $D_{1}$ are still given by (5) with $D_{0}$ $=1$.

The character of the approximations of $u$ to $a$ with the increase of $n$ can be easily observed in the following two cases: 
1. If $v$ and $x$ are not separated by any root of the equation $f(x)=0$, then $u$ and $v$, and also $u$ and $x$, will be separated by the root $a$ alone, provided $n$ is sufficiently large. Therefore $u$ will approach $a$ from the side opposite to $x$ with the increase of $n$. Formula (12) represents a particular case of this rule.

2. If $v$ and $x$ are separated by the root $a$ alone, then $u$ and $v$ will be separated by the root $a$ alone and there will be no root between $u$ and $x$, provided $n$ is large enough. Therefore $u$ will approach $a$ from the side of $x$.

A number of similar results could be obtained by the method employed above. We present below one of them without going into the details of its derivation.

$$
\begin{aligned}
u= & \frac{x-f\left[(v-x)^{2} D_{n-1}+2(v-x) f D_{n-2}+f^{2} D_{n-3}\right]}{\left[(v-x)^{2} D_{n}+2(v-x) f D_{n-1}+f^{2} D_{n-2}\right]} \quad n=5,7, \ldots \\
a= & \frac{x-\lim _{n \rightarrow \infty} f\left[(v-x)^{2} D_{n-1}+2(v-x) f D_{n-2}+f^{2} D_{n-3}\right]}{\left[(v-x)^{2} D_{n}+2(v-x) f D_{n-1}+f^{2} D_{n-2}\right]}
\end{aligned}
$$

In this case $u$ and $x$ will be separated by at least one root of the equation $f(x)=0$ with $v$ an arbitrary real number. Consequently $u$ will approach $a$ from the side opposite to $x$ starting with $n$ sufficiently large.

\section{REFERENCES}

1. E. Bodewig, Sur la méthode de Laguerre pour l'approximation de racines de certaines équations algébriques et sur la critique d' Hermite, Koninklijke Nederlandsche Akademie Wetenschappen Proceedings, XLIX, No. 8 (1946) p. 911-921.

2. E. N. Laguerre, Oeuvres de Laguerre 1, (1898) p. 101 and p. 461.

University of South Carolina 